\title{
Lower Bounds for the Partial Grundy Number of the Lexicographic Product of Graphs
}

\author{
Kenny Domingues, Yuri Silva de Oliveira, Ana Silva \\ ${ }^{1}$ ParGO Group - Parallelism, Graphs and Optimization \\ Centro de Ciências - Departamento de Matemática \\ Universidade Federal do Ceará (UFC)
}

\{kennywille, yurisilva\}ealu.ufc.br, anasilva@mat.ufc.br

\begin{abstract}
A Grundy coloring of a graph $G$ is a coloring obtained by applying the greedy algorithm according to some order of the vertices of $G$. The Grundy number of $G$ is then the largest $k$ such that $G$ has a greedy coloring with $k$ colors. A partial Grundy coloring is a coloring where each color class contains at least one greedily colored vertex, and the partial Grundy number of $G$ is the largest $k$ for which $G$ has a partial greedy coloring. In this article, we give some results on the partial Grundy number of the lexicographic product of graphs, drawing a parallel with known results for the Grundy number.
\end{abstract}

\section{Introduction}

The definitions and notations used in this paper are standard and can be found in any graph theory book. Also, we consider only simple graphs. Graph coloring problems are perhaps the most studied problems in graph theory, due to their practical and theoretical importance. A independent set in a graph $G$ is a subset $S \subseteq V(G)$ such that $u v \notin E(G)$ for every $u, v \in S$. A proper $k$-coloring (from now on called simply a coloring) of a graph $G$ is a partition of $V(G)$ into $k$ independent sets, and the chromatic number of $G$ is the minimum value $\chi(G)$ for which $G$ has a proper $k$-coloring. It is well-known that deciding whether $\chi(G) \leq k$ is NP-complete for every fixed $k \geq 3$ [Karp 1972]. Due to the difficulty of this problem, an alternative is to apply heuristics to solve it. Many heuristics give rise to parameters that study their worst case scenario. In this paper, we focus on a variation of the greedy heuristic.

A coloring obtained with the application of the greedy heuristic is called a Grundy coloring, and the largest integer $k$ for which $G$ has a Grundy coloring is called the Grundy number of $G$ and is denoted by $\Gamma(G)$. Another way of defining such a coloring is as follows. Given a $k$-coloring $S_{1}, \cdots, S_{k}$ of $G$, a vertex in $S_{i}$ is said to be greedy if it has neighbors in $S_{j}$, for every $j<i$. Thus, a Grundy coloring can be seen as a coloring where every vertex is greedy. In constrast, a partial Grundy coloring is defined as a coloring in which each color class has some greedy vertex; the largest $k$ for which $G$ has a partial Grundy coloring is called the partial Grundy number of $G$ and is denoted by $\partial \Gamma(G)$. These parameters were defined in [Christen and Selkow 1979, Erdôs et al. 2003], and while Grundy colorings have been extensively studied, a lot is still to be learned about partial Grundy colorings. In this paper, we investigate partial Grundy colorings of lexicographic products, drawing a parallel with known results for Grundy colorings. 
Before we proceed, we give the formal definition of the lexicographic product. Given graphs $G$ and $H$, the lexicographic product of $G$ and $H$, denoted by $G[H]$, is the graph with vertex set $V(G) \times V(H)$ and the following edge set:

$$
E(G[H])=\{(a, x)(b, y) \mid \text { either } a b \in E(G) \text { or } a=b \text { and } x y \in E(H)\} .
$$

The most basic result concerning the Grundy number of the lexicographic product is the following.

Proposition 1 ([Asté et al. 2010]) Let $G, H$ be graphs. Then $\Gamma(G[H]) \geq \Gamma(G) \Gamma(H)$.

We have found that the analogous result also holds for partial Grundy colorings.

Proposition 2 Let $G, H$ be two graphs. Then $\partial \Gamma(G[H]) \geq \partial \Gamma(G) \partial \Gamma(H)$.

The same authors also proved that the above lower bound is tight. In particular, they show that it is always tight if $G$ is either a tree or satisfies $\Gamma(G)=\Delta(G)+1$.

Theorem 1 ([Asté et al. 2010]) Let $G, H$ be any graphs. If $G$ is either a tree or $\Gamma(G)=$ $\Delta(G)+1$, then $\Gamma(G[H])=\Gamma(G) \Gamma(H)$.

In [Asté et al. 2010], they also give the following upper bound for $\Gamma(G[H])$.

Theorem 2 ([Asté et al. 2010]) $\Gamma(G[H]) \leq 2^{\Gamma(G)-1}(\Gamma(H)-1)+\Gamma(G)$.

Here, we give a lower bound for $\partial \Gamma(G[H])$ better than the one in Proposition 2, and as a consequence, we will get that $\partial \Gamma(G[H])$ can be arbitrarily larger than $\partial \Gamma(G) \partial \Gamma(H)$, and also that no upper bound like the one in the above theorem is possible. For this, we first need some definitions.

Given a graph $G$, a matching in $G$ is a subset $M \subseteq E(G)$ such that $e$ and $e^{\prime}$ share no endpoint, for every $e, e^{\prime} \in M, e \neq e^{\prime}$. We denote the set of endpoints of edges in $M$ by $V(M)$, and we say that a matching is induced if $G$ induced by $V(M)$ contains exactly the edges in $M$. The size of a maximum induced matching in $G$ is called the induced matching number of $G$ and is denoted by $\alpha^{\prime \prime}(G)$.

Theorem 3 Let $G, H$ be graphs. Then $\partial \Gamma(G[H]) \geq(a+1) \partial \Gamma(H)$, where $a=$ $\min \left\{\alpha^{\prime \prime}(G),\left\lfloor\frac{|V(H)|}{\partial \Gamma(H)}\right\rfloor\right\}$.

Observe that if $G$ is itself an induced matching, then $\partial \Gamma(G)=2$, while $\alpha^{\prime \prime}(G)=$ $|V(G)| / 2$. This gives us a hint of why the following holds.

Corollary 1 For every pair of positive integers $2 \leq k \leq \ell$, there exists a path $T$ and a graph $G$ with $\partial \Gamma(T)=2$ and $\partial \Gamma(G)=k$ such that $\partial \Gamma(T[G]) \geq \ell$. The same holds if we take $T$ to be a forest containing only an induced matching instead.

Observe that the above theorem tells us that no upper bound for $\partial \Gamma(G[H])$ exists as a function of $\partial \Gamma(G)$ and $\partial \Gamma(H)$ (like the one in Theorem 2), even if we apply the same constraints as they applied in Theorem 1.

A classic result on the chromatic number of the lexicographic product says that if $\chi(H)=k$ then $\chi(G[H])=\chi\left(G\left[K_{k}\right]\right)$ for every graph $G$ [Geller and Stahl 1975]. The analogous result holds for the Grundy number. 
Theorem 4 ([Asté et al. 2010]) Let $H$ be a graph such that $\Gamma(H)=k$. Then for any graph $G$, we have $\Gamma(G[H])=\Gamma\left(G\left[K_{k}\right]\right)$.

Even though $\partial \Gamma\left(G\left[K_{k}\right]\right)$ is also a lower bound for $\partial \Gamma(G[H])$ (as stated in the next theorem), it can again be arbitrarily bad. To see this, consider $S_{p+1}$ to be the graph on $p+1$ vertices containing no edges, and let $K_{1, p}$ be the tree on $p+1$ vertices containing a vertex, $u$, which is adjacent to all the other vertices (this is also called a star with center $u$ ). Clearly, the graph $G=S_{p+1}\left[K_{1, p}\right]$ consists of $p+1$ disjoint stars isomorphic to $K_{1, p}$; denote by $u_{i}$ the center of the $i$-th star. One can then obtain a partial Grundy coloring of $G$ with $p+1$ colors simply by giving color $i$ to $u_{i}$, and coloring the leaves of the star with center $u_{i}$ with colors $[p+1] \backslash\{i\}$. On the other hand, observe that $\partial \Gamma\left(K_{1, p}\right)=2$, and that $S_{p+1}\left[K_{2}\right]$ is simply an induced matching on $2(p+1)$ vertices, and hence $\partial \Gamma\left(S_{p+1}\left[K_{2}\right]\right)=$ 2. Therefore, for every $p \geq 2$, we get $\partial \Gamma\left(S_{p+1}\left[K_{1, p}\right]\right) \geq p+1>2=\partial \Gamma\left(S_{p+1}\left[K_{k}\right]\right)$, where $k=\partial \Gamma\left(K_{1, p}\right)$. Nevertheless, as we previously said, the value is still a lower bound.

Theorem 5 Let $H$ be a graph such that $\partial \Gamma(H)=k$. Then for any graph $G$, we have $\partial \Gamma(G[H]) \geq \partial \Gamma\left(G\left[K_{k}\right]\right)$.

Up to our knowledge, there are no further results about the Grundy number of the lexicographic product of graphs. Because of space constraints, we present only some of our proofs in the next section. In the lexicographic product $G[H]$, for each vertex $x \in V(G)$, we call copy of $H$ in $x$ the subgraph of $G[H]$ induced by the vertices $\{x\} \times$ $V(H) \subseteq V(G[H])$, and we denote it by $x[H]$. Clearly $x[H]$ is isomorphic to $H$. Note also that the lexicographic product is not commutative, unlike other graph products.

\section{Outline of the proofs}

A $k$-coloring can also be defined as a function $f$ of $V(G)$ into $[k]$ such that $f(u) \neq f(v)$ for every $u v \in E(G)$. The analogous definitions of Grundy coloring and partial Grundy coloring are straightforward. Let $f, f^{\prime}$ be colorings of a graph $H$. We say that $f^{\prime}$ is obtained from $f$ by a shift of $t$ if $f^{\prime}(u)=f(u)+t$ for every $u \in V(H)$.

We ommit the proof of Proposition 2 since it is quite simple. It suffices to take partial Grundy colorings $f, f^{\prime}$ of $G$ and $H$, respectively, and color $(u, x) \in V(G[H])$ with $\left(f(u), f^{\prime}(x)\right)$.

Proof of Theorem 3: Let $a=\min \left\{\alpha^{\prime \prime}(G),\left\lfloor\frac{\lfloor V(H) \mid}{\partial \Gamma(H)}\right\rfloor\right\}$, and let $M=$ $\left\{u_{1} v_{1}, \ldots, u_{a} v_{a}\right\}$ be an induced matching of size $a$ in $G$; it exists since $a \leq \alpha^{\prime \prime}(G)$. Also, let $f$ be a partial Grundy coloring of $H$ with $k=\partial \Gamma(H)$ colors. We construct a partial Grundy coloring $f^{\prime}$ of $G[H]$ with at least $(a+1) k$ colors. The idea is to make the copy $v_{i}[H]$ contain greedy vertices of colors $\{i k+1, \cdots,(i+1) k\}$, while the greedy vertices of colors $\{1, \cdots, k\}$ are in the copy $u_{1}[H]$. For this, we color $u_{1}[H]$ exactly as $f$ and, for each $i \in\{1, \ldots, a\}$, let $f^{\prime}$ restricted to $v_{i}[H]$ be obtained from $f$ by a shift of $i k$; therefore, the colors used in $v_{i}[H]$, as previously said, are exactly $\{i k+1, \ldots,(i+1) k\}$. It remains now to ensure that the vertices in $v_{i}[H]$ are also adjacent to colors $\{1, \cdots, i k\}$. This already holds for $i=1$, and to ensure that it holds for higher values of $i$, we simply color the copy $u_{i}[H]$ with any proper coloring of $H$ that use the colors $\{1, \ldots, i k\}$, for each $i \in\{2, \ldots, a\}$. Observe that this is possible since, because $a \leq\left\lfloor\frac{|V(G)|}{\partial \Gamma(H)}\right\rfloor$, we get $|V(H)| \geq a k \geq i k$; hence it suffices to start with say $f$, and add new colors until we reach the desired coloring. Now we have that $u_{i}[H]$ contains all colors in $\{1, \ldots, i k\}$ and 
$v_{i}[H]$ is complete for $u_{i}[H]$. It can be seen that the obtained coloring $f^{\prime}$ contains greedy vertices of all colors in $\{1, \ldots,(a+1) k\}$. The remaining vertices can be colored greedily to obtain a partial greedy coloring with at least the same number of colors as $f^{\prime}$.

Proof of Corollary 1: Let $a$ be any value such that $(a+1) k \geq \ell$, and let $T=P_{n}$, where $n=3 a+2$. It is easy to verify that $\alpha^{\prime \prime}(T)=a$. Also, let $G$ be a graph with $\partial \Gamma(G)=k$ and at least $a$ vertices; for instance, we can take the complete graph on $k$ vertices, and add isolated vertices if necessary in case $a>k$. By Theorem 3, we get directly that $\partial \Gamma(T[G]) \geq(a+1) k \geq \ell$. Finally, the same clearly holds if, instead of $T=P_{n}$, we take $T$ on $2 a$ vertices containing exactly a perfect matching.

\section{Conclusion}

In this paper, we have studied the partial Grundy number of the lexicographic product $G[H]$. We have given a lower bound as a function of a maximum induced matching of $G$ and the partial Grundy number of $H$, getting as a result that no upper bound as function of $\partial \Gamma(G)$ and $\partial \Gamma(H)$ can exist, in contrast with the Grundy number. We also proved that $\partial \Gamma(G[H]) \geq \partial \Gamma\left(G\left[K_{k}\right]\right)$, where $k=\partial \Gamma(H)$, while getting yet as a consequence of the previous result that this lower bound can be arbitrarily bad. A good question is whether the lower bound obtained in Theorem 3 is asymptotically best possible.

Question 1 Given graph $G$ and $H$, does there exist an upper bound on $\partial \Gamma(G[H])$ as a function of $\alpha^{\prime \prime}(G)$ and $\partial \Gamma(H)$ ?

In particular, in [Shi et al. 2005] the authors give exact values for the partial Grundy number of trees. Hence it would be interesting to answer Question 1 when $G$ is a tree.

Additionally, other graph products should be investigated, namely the direct product $G \times H$, the cartesian product $G \square H$ and the strong product $G \otimes H$. Up to our knowledge, none of them has been investigated yet.

\section{References}

Asté, M., Havet, F., and Linhares-Sales, C. (2010). Grundy number and products of graphs. Discrete Mathematics, 310(9):1482-1490.

Christen, C. A. and Selkow, S. M. (1979). Some perfect coloring properties of graphs. Journal of Combinatorial Theory, Series B, 27(1):49-59.

Erdős, P., Hedetniemi, S. T., Laskar, R. C., and Prins, G. C. (2003). On the equality of the partial grundy and upper ochromatic numbers of graphs. Discrete Mathematics, 272(1):53-64.

Geller, D. and Stahl, S. (1975). The chromatic number and other functions of the lexicographic product. Journal of Combinatorial Theory, Series B, 19(1):87-95.

Karp, R. M. (1972). Reducibility among Combinatorial problems, pages 85-103. Springer US.

Shi, Z., Goddard, W., Hedetniemi, S. T., Kennedy, K., Laskar, R., and McRae, A. (2005). An algorithm for partial grundy number on trees. Discrete Mathematics, 304(1-3):108116. 